\title{
Heart Diseases Diagnosis Using Intelligent Algorithm Based on PCG Signal Analysis
}

\author{
Mohammed Nabih-Ali' ${ }^{1}$, El-Sayed A. El-Dahshan ${ }^{1,2}$, Ashraf S. Yahia ${ }^{2}$ \\ ${ }^{1}$ Faculty of Computer and Information Technology, Egyptian E-Learning University (EELU), Giza, Egypt \\ ${ }^{2}$ Physics Department, Faculty of Sciences, Ain Shams University, Cairo, Egypt \\ Email:mnabih@eelu.edu.eg, seldahshan@eelu.edu.eg,ayahia@sci.asu.edu.eg
}

How to cite this paper: Nabih-Ali, M., El-Dahshan, E.-S.A. and Yahia, A.S. (2017) Heart Diseases Diagnosis Using Intelligent Algorithm Based on PCG Signal Analysis. Circuits and Systems, 8, 184-190. https://doi.org/10.4236/cs.2017.87012

Received: January 12, 2017

Accepted: July 25, 2017

Published: July 28, 2017

Copyright (C) 2017 by authors and Scientific Research Publishing Inc. This work is licensed under the Creative Commons Attribution International License (CC BY 4.0).

http://creativecommons.org/licenses/by/4.0/

Open Access

\begin{abstract}
This paper presents an intelligent algorithm for heart diseases diagnosis using phonocardiogram (PCG). The proposed technique consists of four stages: Data acquisition, pre-processing, feature extraction and classification. PASCAL heart sound database is used in this research. The second stage concerns with removing noise and artifacts from the PCG signals. Feature extraction stage is carried out using discrete wavelet transform (DWT). Finally, artificial neural network (ANN) has been used for classification stage with an overall accuracy $97 \%$.
\end{abstract}

\section{Keywords}

Heart Diseases, Phonocardiogram (PCG), Feature Extraction, Discrete Wavelet Transform (DWT), Artificial Neural Network (ANN)

\section{Introduction}

Cardiovascular disorders (CVDs) or Heart diseases are broad terms that can affect both vasculature and the heart muscle itself [1]. CVDs remains the first cause of mortality globally, responsible for 17.5 million people dying annually [2]. More than $75 \%$ of CVDs deaths occur in middle and low income countries [2].

The heart sounds are still the primary tool for screening and diagnosing many pathological conditions of the human heart. Using auscultation technique which was invented and defined by Laennec for heart sound analysis is still insufficient. The reason of insufficiency is reported by Avendano-Valencia et al., [3] [4] due to the human ear limitation and subjective of the analyst and the discriminatory skills that can take many years to acquire.

Phonocardiography (PCG) is one of non-invasive techniques to diagnose 
condition of human heart generated by muscle contractions and closure of the heart valves produces vibrations audible as sounds and murmurs, which can be analyzed by qualified cardiologists [5].

The PCG recording consists of four heart sound components (S1, S2, S3 and S4). The first and second heart sound (S1 and S2) can be heard from the normal heart. They are produced by the closure and opening of the normal valves. For the abnormal heart, a third and a fourth sound (S3 and S4) may also exist in addition to (S1) and (S2) [6]. These abnormal sounds are called murmurs. The existing of murmur in PCG recording is often related to heart valve disease. The four heart sounds (S1, S2, S3 and S4) are shown in Figure 1.

A major challenge, facing healthcare organizations (hospitals, medical centers) is the provision of quality services at affordable costs. Quality service implies diagnosing patients correctly and administering treatments that are effective [7].

Several researches, various methodologies and many tools that had been proposed were used in recently published papers for medical systems development for heart diseases diagnosis [8] [9] [10]. Unfortunately, the achieved accuracy was not satisfactory so, the work is devoted to proposing an algorithm for heart diseases diagnosis with high accuracy.

The objective of this paper is to propose an intelligent algorithm based on PCG signals to integrate the clinical decision support with computer-based patient records. This could reduce medical errors, enhance patient safety, decrease unwanted practice variation, and improve patient outcome.

This paper is organized as follows. Section 2 presents the proposed machine learning techniques and the aspects of data acquisition, pre-processing, feature extraction and classification processes. In Section 3, experimental results are shown. Finally, Section 4 concludes the paper and proposes future research work.

\section{Proposed Methodology}

A methodology of a heart diseases diagnosis system usually mimics that of a

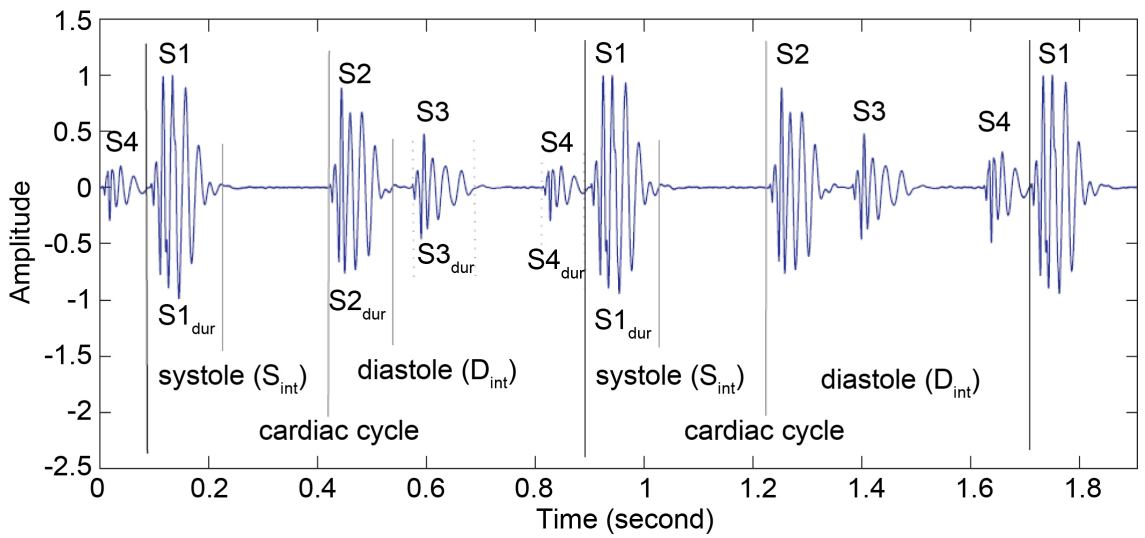

Figure 1. PCG heart sound signal. 
pattern recognition system. Thus, it can be broken down into four main processes, namely; 1) data acquisition, 2) pre-processing; 3) feature extraction and 4) classification (subject identification). Figure 2 shows the proposed methodology for heart diseases diagnosis. In the following subsection each stage will be discussed.

The experiments were carried out on the platform of core i3 with $3 \mathrm{G}$ memory running under windows 1064 bit. The proposed algorithms were developed using MATLAB 2014b wavelet toolbox.

\subsection{Data Acquisition}

Datasets were collected from PASCAL heart sound database [11]. A data base of 170 heart sound signals was created and divided into 121 signals (24 normal signals and 97 abnormal signals) for training and 49 signals (10 normal signals and 39 abnormal signals).

The acoustic audio files have varying lengths, between 1 second and 30 seconds. Mainly the most information in heart sounds is enclosed in the low frequency components, having noise in the higher frequencies.

\subsection{Signal Pre-Processing}

PCG signals usually suffers from noise like electromagnetic interference from surrounding environment, power frequency interference, electrical signal inter-

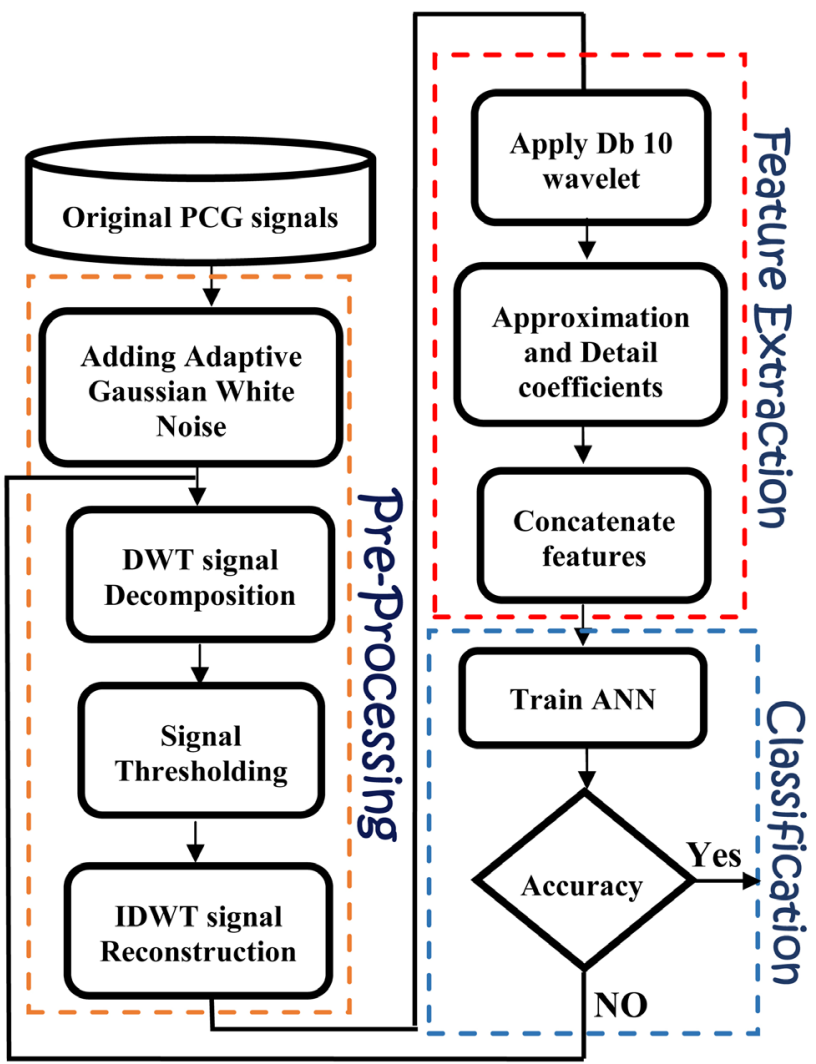

Figure 2. Proposed methodology. 
ference with human body and lung sounds. These various noise components make the diagnosis of PCG records difficult or in some cases is impossible.

DWT which is a series of high-pass and low-pass filters shows a superior performance in signal denoising due to its properties such as multi-resolution and windowing technique.

Noises in PCG signals was removed using DWT family called Daubechies wavelet family of order ten ( $\mathrm{Db} 10)$ with the fourth level of decomposition. Figure 3 shows the noisy PCG signal and the denoised PCG signal.

\subsection{Feature Extraction}

Feature extraction is one of the most important steps of heart sound classification systems [12]. Time-frequency domain wavelet based feature extraction technique has been successfully used to extract important features from non-stationary heart sound signals for heart diseases classification.

The spectrum of heart sound signals is divided into sub-bands (tree structure was used) to extract the most meaningful information from normal and abnormal heart sounds.

Daubechies wavelet with $4^{\text {th }}$ order $(\mathrm{Db} 4)$ with $10^{\text {th }}$ level of decomposition is used for determining wavelet coefficients from the PCG signals. DWT decomposes the signal into approximation (Approximate coefficients (A)-low frequency coefficients of signal) and detail (Detail coefficients (D)-high frequency part components of signal).

(a) Noisy PCG Signal

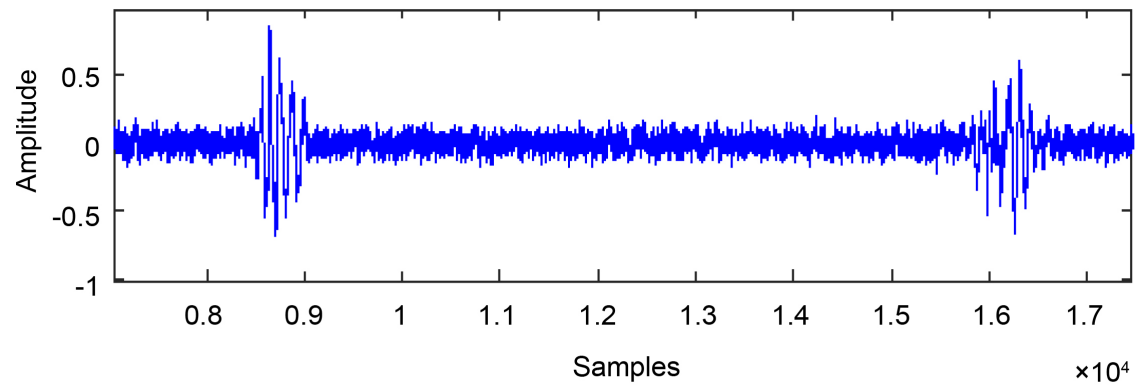

(b) De-noised PCG Signal

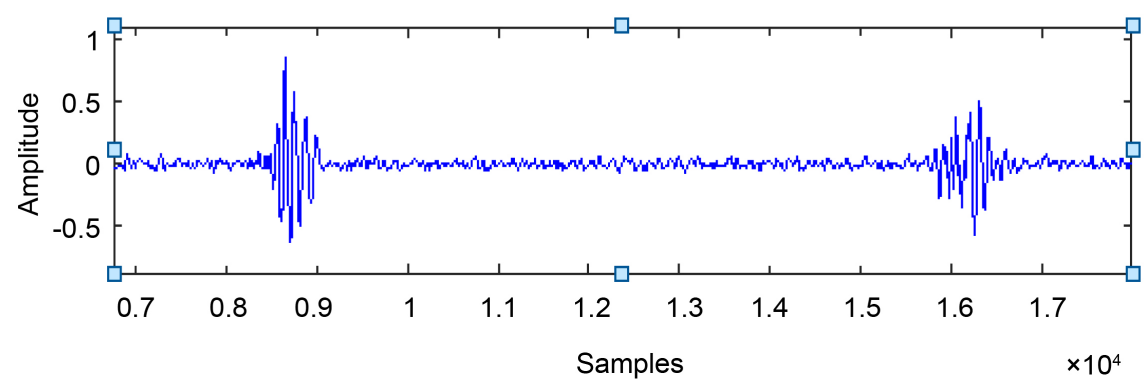

Figure 3. (a) Noisy PCG signal (b) de-noised PCG signal. 


\subsection{Classification}

The classification process of the artificial neural network begins with sum of multiplication of weights and inputs plus bias at the neuron, if the sum is positive then only output elements fires. Otherwise it doesn't fire. The artificial neural network is an adaptive system, in other words, the system adopting itself and changes the system weights during the operation [13].

The feature vector extracted from the PCG signal with size 44 features is used as an input to the proposed neural network to classify it into 2 classes (normal and abnormal). The proposed neural network architecture consists of three layers input layer with 44 input neurons, one hidden layer with 10 neurons and one output layer with 2 neurons. Figure 4 shows the architecture of the proposed ANN.

Levenverg-Marquardt back propagation algorithm is used for neural network training, sigmoid activation function, momentum constant equal 0.7, learning rate 0.9 and number of epochs 1000 iterations. Table 1 shows the parameters used for training the neural network classifier.

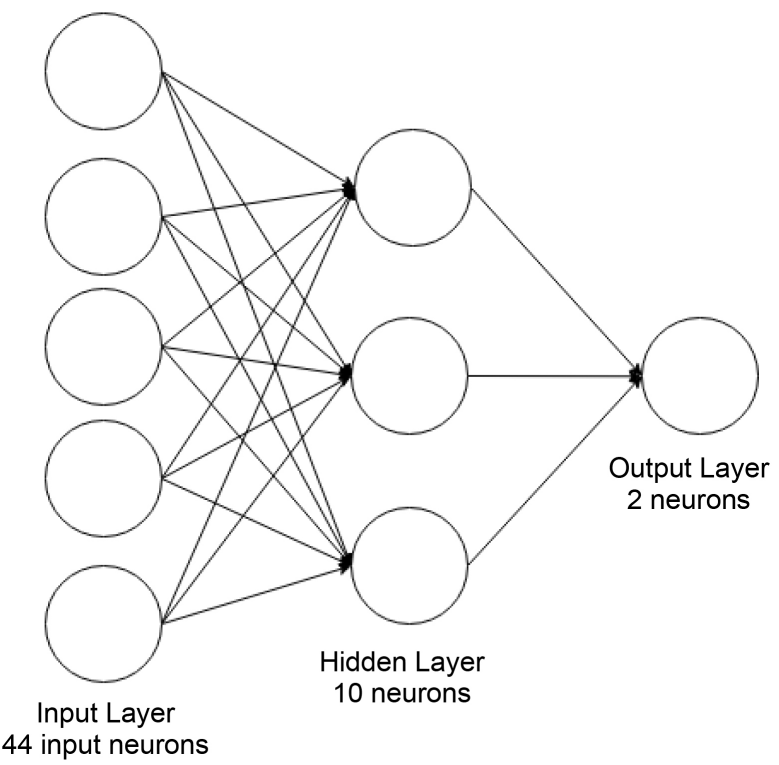

Figure 4. Proposed artificial neural network.

Table 1. Proposed Neural network training parameters.

\begin{tabular}{cc}
\hline Network Type & Feed-forward back propagation \\
\hline No. of layers & 3 layers: input, hidden and output layers \\
Activation function & Sigmoid activation function \\
Training algorithm & Levenverg-Marquardt back propagation \\
No. of epochs & 1000 \\
Accuracy & $97 \%$ \\
Momentum constant & 0.7 \\
Learning rate & 0.9 \\
\hline
\end{tabular}


Table 2. Comparison between our proposed methodology and previous proposed methodologies.

\begin{tabular}{cccc}
\hline Author & Database & Methods & Results \\
\hline $\begin{array}{c}\text { Mandeep Singh } \\
\text { (2013) [14] }\end{array}$ & PASCAL dataset & Naïve Bayes classifier & Accuracy \\
Elsa Ferreira & PASCAL & Decision tree classification & $93.33 \%$ \\
(2013) [15] & dataset & algorithm & Accuracy \\
N. R. Sujit & PASCAL & Regression Tree & $72.76 \%$ \\
(2016) [16] & dataset & & Accuracy \\
Zichun Tong & PASCAL & Hilbert Transform + Support & $78.33 \%$ \\
(2015) [17] & dataset & vector machine & Accuracy \\
& & & $90.5 \%$ \\
Our proposed System & PASCAL dataset & DWT and ANN & Accuracy \\
& & & $97 \%$ \\
\hline
\end{tabular}

For 170 heart sound signals was created and divided into 121 signals (24 normal signals and 97 abnormal signals) for training and 49 signals (10 normal signals and 39 abnormal signals) the neural network classifier misclassified one signal achieving an accuracy of $97 \%$.

Finally, Table 2 shows a comparison between our proposed algorithm results with the previous methodologies results to see the efficiency of the proposed methodology.

From Table 2, it is clear that our proposed algorithm reached better classification accuracy than the compared studies.

\section{Conclusions}

This paper proposed an algorithm for heart diseases diagnosis based on PCG signals. The proposed algorithm consists of four stages: Data acquisition, preprocessing, feature extraction and classification. PASCAL PCG signal database was used for training and testing the proposed algorithm. We applied DWT in noise elimination and feature extraction stages and neural network is used for PCG signals classification. A feature vector of size 44 features is formed using DWT used as an input to the classifier for training and testing. ANN is trained using the obtained features. The results showed accuracy of $97 \%$ for PASCAL heart sound database.

Despite the high accuracy obtained, the system still has some drawbacks like using small dataset, existence of low energy events or noise and the limitation of classifier used for detecting and diagnosis of heart diseases can affect on the diagnosing accuracy. In the future work we can mention that working towards hybrid techniques which hybrids more than one classification techniques to achieve more accurate results.

\section{References}

[1] Salem, A.-B.M., Revett, K. and El-Dahshan, E.-S.A. (2009) Machine learning in electrocardiogram diagnosis. International Multiconference on Computer Science 
and Information Technology. IEEE, 429-433.

https://doi.org/10.1109/imcsit.2009.5352689

[2] http://www.who.int/cardiovascular_diseases/en/

[3] Reed, T.R., Reed, N.E. and Fritzson, P. (2003) Heart Sound Analysis for Symptom Detection and Computer-Aided Diagnosis. Simulation: Modelling Practice and Theory, 12, 129-146. https://doi.org/10.1016/j.simpat.2003.11.005

[4] Avendaňo-Valencia, L.D., Ferrero, J.M. and Castellanos-Dominguez, G. (2007) Improved Parametric Estimation of Time-Frequency Representation for Cardiac Murmur Discrimination. Computers in Cardiology, 35, 157-160.

[5] Abbas, A.K., Bassam, R. and Kasim, R.M. (2008) Mitral Regurgitation PCG-Signal Classification Based on Adaptive Db-Wavelet. 4th Kuala Lumpur International Conference on Biomedical Engineering, Springer Berlin Heidelberg, 212-216.

[6] Roy, A.K., Misal, A. and Sinha, G.R. (2014) Classification of PCG Signals: A Survey.

[7] Mann, D.L., et al. (2014) Braunwald's Heart Disease: A Textbook of Cardiovascular Medicine. Elsevier Health Sciences.

[8] Sivagowry, S., Durairaj, M. and Persia, A. (2013) An Empirical Study on Applying Data Mining Techniques for the Analysis and Prediction of Heart Disease. 2013 International Conference on Information Communication and Embedded Systems (ICICES), IEEE. https://doi.org/10.1109/icices.2013.6508204

[9] Dokur, Z. and Ölmez. T. (2008) Heart Sound Classification Using Wavelet Transform and Incremental Self-Organizing Map. Digital Signal Processing, 18, 951-959. https://doi.org/10.1016/j.dsp.2008.06.001

[10] Kao, W.-C. and Wei, C.-C. (2011) Automatic Phonocardiograph Signal Analysis for Detecting Heart Valve Disorders. Expert Systems with Applications, 38, 6458-6468. https://doi.org/10.1016/j.eswa.2010.11.100

[11] http://www.peterjbentley.com/heartchallenge/

[12] Hanbay, D. (2009) An Expert System Based on Least Square Support Vector Machines for Diagnosis of the Valvular Heart Disease. Expert Systems with Applications, 36, 4232-4238.

[13] Haykin, S. (1999) Neural Networks: A Comprehensive Foundation. Prentice Hall, Upper Saddle River.

[14] Singh, M. and Amandeep, C. (2013) Heart Sounds Classification Using Feature Extraction of Phonocardiography Signal. International Journal of Computer Applications, 77, 13-17. https://doi.org/10.5120/13381-1001

[15] Gomes, E.F., Jorge, A.M. and Azevedo, P.J. (2013) Classifying Heart Sounds Using Multi-Resolution Time Series Motifs: An Exploratory Study. Proceedings of the International Conference on Computer Science and Software Engineering, Porto, 10-12 July 2013, 23-30. https://doi.org/10.1145/2494444.2494458

[16] Sujit, N.R., Kumar, C.S. and Rajesh, C.B. (2016) Improving the Performance of Cardiac Abnormality Detection from PCG Signal. Advancement in Science and Technology: Proceedings of the 2nd International Conference on Communication Systems, 1715. https://doi.org/10.1063/1.4942735

[17] Tong, Z. (2015) An Integrated Framework for Cardiac Sounds Diagnosis. 
Submit or recommend next manuscript to SCIRP and we will provide best service for you:

Accepting pre-submission inquiries through Email, Facebook, LinkedIn, Twitter, etc. A wide selection of journals (inclusive of 9 subjects, more than 200 journals)

Providing 24-hour high-quality service

User-friendly online submission system

Fair and swift peer-review system

Efficient typesetting and proofreading procedure

Display of the result of downloads and visits, as well as the number of cited articles Maximum dissemination of your research work

Submit your manuscript at: http://papersubmission.scirp.org/

Or contact cs@scirp.org 